\title{
Cosmic Background
}

Chairman \& Editor: R.B. Partridge 


\title{
OPENING REMARKS
}

\author{
R. B. Partridge \\ Haverford college \\ Haverford, PA 1904]
}

The following papers formed a Joint Discussion sponsored by Commissions $40,44,47$ and 48 of the International Astronomical Union. The topic was Cosmic Backgrounds. The plural "backgrounds" is important. Speakers considered electromagnetic radiation fields ranging from radio wavelengths to $\mathrm{X}$-rays. Because other sessions at this General Assembly treated higher energy backgrounds, in particular the $\mathrm{X}$-ray region, emphasis in this Joint Discussion was on radio and infrared backgrounds. In addition to the wide range of wavelengths covered, the session also dealt with a wide range of models for various backgrounds. For instance, at some wavelengths (e.g., $\lambda \sim 1 \mathrm{~cm}$ ) the background is truly a background, generated at very high redshifts--in this case a thermal radiation field at $\sim 3 \mathrm{~K}$. At other wavelengths (e.g., in the X-ray region) the "background" may consist in whole or in part of the summed emission from nany unresolved or subliminal sources.

In addition to papers of moderate length (4-10 pages), there are a number of shorter contributions here. These represent both short comments delivered orally and several poster papers associated with this Joint Discussion. It is with regret that $I$ have to add that Jeremiah ostriker, who was to give one of the major talks, was unable at the last minute to attend the Joint Discussion.

Let me end with thanks to the Commissions which agreed to sponsor this Joint Discussion and to all those who participated in it. 